
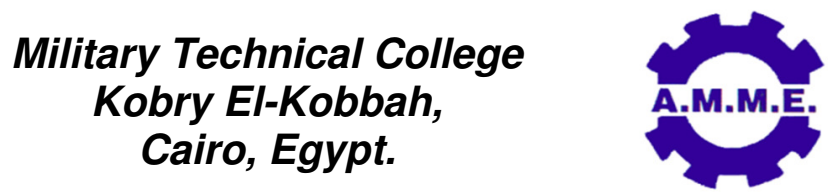

\title{
DETERMINING MATERIAL CONSTITUTIVE PARAMETERS ON STEEL SHEETS
}

\author{
M.A.G. Calle and M. Alves ${ }^{*}$
}

\begin{abstract}
Finite element analysis is extensively employed to predict the structural response of mechanical components so reducing costs of experimental programs. However, among other aspects, the accuracy of the input material parameters in the finite element modeling strongly determines the simulation accuracy. The aim of this study is to evaluate the material constitutive parameters, considering an elasto plastic material model with strain rate sensitivity and failure and by using an inverse methodology. The evaluated material was a mild steel sheet used in scale models of naval structures. Quasi static and dynamic tests were performed and the so obtained material parameters were corroborated through numerical modelings of notched and shear specimens tests. Finally, a discussion about the evaluation of strain rate sensitivity model and failure criteria parameters is also presented.
\end{abstract}

\section{KEY WORDS}

Material characterization, strain rate sensitivity, failure, steel sheet

* Dr. Eng., Department of Mechatronics and Mechanical Systems, Polytechnic School of the University of Sao Paulo, Sao Paulo, Brazil.

** Professor Dr. Eng., Department of Mechatronics and Mechanical Systems, Polytechnic School of the University of Sao Paulo, Sao Paulo, Brazil. 


\section{INTRODUCTION}

Most catastrophic accidents with ships occur due to collision and grounding. The risk of ship collision had increased with the growth of the global fleet of ships. The injury caused by a ship collision not only causes oil spill and ship structure damage but also leads to degradation of the marine environment, explosions, human losses, blocking of ships traffic and permanent damage to the ship [1]. This global scenario emphasizes the necessity of reformulating the security in the entire offshore oil exploration chain, as well as investing in better ship designs and a better knowledge of the material behavior for ship construction.

Models in reduced scale are important in naval engineering since the experimental test of real size ships can be very expensive and require large testing machines. However, only hydrodynamic ship aspects are traditionally evaluated by scaled models. The study of the structural behavior of ships during a ship collision event using reduced scale model is not a common study in the ship research community [2-3]. The large quantity of structural details of a real ship structure is one of the principal difficulties to overcome when working in a reduced scale [2]. When opting for a finite element modeling of the collision, it is imperative an adequate mechanical characterization of the structural material [4]. During an impact event of a thin plate structure, both force-displacement response and structural collapse mode are strongly dependent of the mechanical behavior of the material.

The main mechanical test employed in industrial applications to obtain the mechanical properties of materials is the uniaxial tensile test. Practical procedures for obtaining the stress strain curve from a uniaxial tensile test are generally standardized for industrial use. These methodologies give valuable information for industrial requirements but are somewhat restricted for scientific investigations. In tensile samples, after necking formation, the information obtained is somewhat limited due to difficulties in measuring experimentally local stresses and strains. Some investigations in the last years overcome these practical limitations by correcting the material curve based in empirical data [5], and improving other more traditional studies about correcting material curve in circular section specimens [6-7]. In recent years, non-contact photogrammetric techniques as the Digital Image Correlation (DIC) became powerful experimental tools for measuring strains field directly in the specimen surface [8]. Due to the large use of sheet material in industry, some researchers studied how to obtain equivalent true stress strain curves from uniaxial tensile tests of sheet samples. These methods are complex to implement because in some cases it requires devices to measure parameters such as width and thickness during the tests [9], or iterative routines based on analytical formulations of the plasticity theory. On the other hand, inverse methods have been used recently in the identifications of material parameters from experimental data using optimization techniques [10].

Other two important points in the numerical analysis of ship collision events are the effect of deformation in high velocities and the material failure. Both have a strong influence on the global structural energy absorption and the fracture extension in structures [1]. Many researches stated the strong influence of the high deformation velocities of steel naval structures in a collision event. The direct consequence of the high deformation velocities is the increase in the material resistance so allowing 
major energy absorption of thin plate structures and achieving major force peaks in the structural response during the impact [11-12]. On the other hand, the assessment of the structural failure in a ship collision event is a very complex process influenced by many factors as the mechanical properties of the structural materials, geometry of the structural members, constructive imperfections, application of the loads, velocity of the loads as many others. These diverse factors can promote different collapse modes normally observed in collided naval structures as cutting, concertina tearing, folding, crushing, ruptured plate and membrane stretching [13]. To reproduce a similar structural response by numerical modelling of a ship collision, an adequate failure criterion should promote most of these collapse modes. Some failure criteria developed specifically for ship collision events took these aspects into account by using relationships between principal stresses or principal strains, as triaxiality and the Lode parameter [14].

In this work, an optimization methodology is presented to obtain the material parameters to define the elasto plastic behavior of a mild steel material using the constitutive model of Voce. The steps to evaluate the parameters of the strain rate sensitivity model from experimental tests were also presented. Finally, the material failure parameters were evaluated in seven different specimen geometries to comprise a wide range of triaxialities. A failure criterion developed specifically for numerical modeling of ship collision events was used and an optimization routine was also necessary to find the failure parameters for each specimen geometry.

\section{ELASTO PLASTIC MATERIAL MODEL}

Mild steel sheets was used in preceding researches [4,15-16] for the construction of reduced scale naval structures due to its similar mechanical properties to the naval steels. In this work, mild steel sheets (SAE 1008), with $0.25 \mathrm{~mm}$ thickness, was used to evaluate the mechanical properties due to its ability to represent better welded naval structures in a reduced scale of 1:100.

Commonly, the elasto plastic behavior of a mild steel material (SAE 1008) can be described by the stress strain curve which can be obtained using a dog bone sample subjected to a tensile test. In the stress strain curve, obtained from a tensile test of mild steel, three stages can be identified during the test: elastic regime, plastic regime before necking and plastic regime after necking to rupture. Standard procedures allow the calculation of the engineering and true strain and stress values from experimental test data, equations (1-4).

$$
\begin{aligned}
& e=\frac{L-L_{0}}{L_{0}}=\frac{\Delta L}{L_{0}} \\
& S=\frac{F}{A_{0}} \\
& \varepsilon=\int_{L_{0}}^{L} \frac{d L}{L}=\ln \left(\frac{L}{L_{0}}\right)=\ln (1+e) \\
& \sigma=\frac{F}{A}=S \frac{A_{0}}{A}=S \frac{L}{L_{0}}=S(1+e)
\end{aligned}
$$


where $S$ and $e$ are the engineering stress and strain, $L_{0}$ and $L$ are the initial and current length of the specimen, $F$ is the force, $A_{0}$ and $A$ are the initial and current section area of the specimen, $\sigma$ and $\varepsilon$ are the true stress and strain.

During the elastic and plastic regimes before necking, the specimen experiences a uniform strain. But after the necking point, the strain evolves just locally in the neck. As a result, the limitations to obtain the plastic behavior of any material using standard uniaxial tensile tests begin when the necking initiates because the strain distribution in the section of the specimen is no longer uniform and the strain history will not represent the real local plastic behavior [17]. Even using any technique for strain acquisition, the estimated curve will be unable to follow the real stress strain curve of the material. Photogrammetric techniques are capable to widen the extent of the strain acquisition, but these techniques are limited to the number of images acquired, minimum element size and the maximum supported distortion of the pattern painted in the specimen surface. On the other hand, the optimization procedures permits to obtain the entire stress strain curve until complete failure thanks to the fine tuning of the material parameters [17].

An inverse methodology was developed for obtaining the material parameters of the analyzed material. This methodology consists in the adjustment of the numerical model response to the analogous experimental response of certain mechanical test, in this case the uniaxial tensile tests. This adjustment is based in the progressive fine tuning of certain parameters of the numerical model to attain the desirable response mentioned above. This fine tuning was achieved using an optimization methodology where the optimization variables are the parameters of the material constitutive model, equation (5), and the objective function is the minimum difference between the force displacement curves obtained from the experimental test and numerical modeling, equation (6). The diagram presented in Figure 1 explains the procedure for adjusting the material variables.

$$
\begin{aligned}
& \sigma=k+R_{0} \cdot \varepsilon_{p}+R_{\infty}\left(1-e^{-b \varepsilon_{p}}\right) \\
& \text { Minimize }\left\{\sum_{i=1}^{n}\left|F_{i}^{\text {exp }}-F_{i}^{\text {num }}\left(R_{0}, R_{\infty}, b\right)\right|\left(\frac{d_{i+1}-d_{i-1}}{2}\right)\right\}
\end{aligned}
$$

where $\varepsilon_{p}$ is the plastic strain, $k$ if the yield stress (fixed directly from the experimental test data), $R_{0}, R_{\infty}$ and $b$ are the variables of the Voce material model which need to be adjusted by the optimization routine, $\left(F_{i}^{\text {exp }}, d_{i}\right)$ and $\left(F_{i}^{\text {num }}, d_{i}\right)$ are the $i$-th point of the force displacement curves obtained experimental and numerically, respectively, from the uniaxial tensile test given that $F_{i}^{\text {num }}$ is dependent on the variables of the Voce material model. The Voce material model was chosen because this material model describes better than other models the plastic behavior because it employs four parameters. The binary search was implemented in the optimization routine because its simplicity and efficacy to find the optimum three parameters by performing a search in the tridimensional space (variables) decreasing the step progressively to achieve the minimum value of the objective function. The optimization routine was programmed in Pascal language and the LS-dyna code was used in the FE model processing in explicit analysis. The 2D FE model of the specimen has dimensions $50 \times 10 \times 0.25 \mathrm{~mm}$ and was created using quadrilateral $0.5 \times 0.5 \mathrm{~mm}$ shell elements considering the Hughes-Liu formulation [18] and five 
integration points through the thickness. The numerical model was created in the Ansys R13 code, the Ls-Dyna 971 was used as numerical solver and the LS-PrePost 4.0 for the post-processing of results. The material failure was not taken into account in the numerical model for the optimization routine.

The experimental force displacement response of a uniaxial tensile test was performed in an Instron universal testing machine, model 3369. The experimental force displacement response was acquired considering the force from the load cell of the machine and the displacement within two points, set apart by $50 \mathrm{~mm}$ and centered in the length of the specimen, which equals the clip gage set up.

The entire optimization process required 53 optimization cycles where each cycle required 5 finite element processes of about 24 min each one. The resulting material parameters were estimated with an accuracy of $0.025 \%$ resulting in $k=219.0 \mathrm{MPa}$, $R_{0}=479.8220 \mathrm{MPa}, R_{\infty}=126.2866 \mathrm{MPa}$ and $b=21.4768$. Both experimental and numerical force displacement curves were presented in Figure 2.

\section{STRAIN RATE SENSITIVITY}

In general, mild steel is very sensitive to high strain rates so showing higher material resistance as straining velocity increases. The Cowper-Symonds model [19] is a traditional and well-known strain rate sensitivity model, easily found in commercial finite element codes, linked to the elasto plastic material model to include the influence on the material resistance due to its sensitivity to strain rates. This model is dependent on two parameters $C$ and $p$ which are obtained experimentally. The dynamic increment factor (DIF), defined as the true stress obtained at dynamic test conditions divided by the true stress under quasi static test conditions is defined by the Cowper-Symonds sensitivity model as shown in equation (7).

$$
\mathrm{DIF}=1+\left(\frac{\dot{\varepsilon}}{c}\right)^{1 / p}
$$

where $\dot{\varepsilon}$ is the effective strain rate. The tests performed to evaluate the CowperSymonds parameters were divided into two groups: low and high velocity tests. So, two testing machines were required to perform the tests. For the low velocity tests, uniaxial tensile tests were performed in an Instron universal testing machine, model 3369 , achieving test velocities from 0.1 to $150 \mathrm{~mm} / \mathrm{min}$ so inducing strain rates in the range from 0.00004 to $0.05 \mathrm{~s}^{-1}$. Flat specimens of $50 \times 10 \times 0.25 \mathrm{~mm}$ dimensions were used. Besides, for the high velocity tests, $12 \mathrm{~mm}$ diameter disc samples were tested in a split Hopkinson pressure bar so resulting in strain rates about $6000 \mathrm{~s}^{-1}$. The Hopkinson bar is the most traditional machine to measure the stress and strain curve at high strain rates. The split Hopkinson bar consists of two slender bars and a specimen mounted between them. By striking the end of the bar, a stress wave is generated so propagating down the bar towards the specimen. The elastic wave partially reflected and transmitted are proportional to the strain rate and the stress of the tested material. Figure 3 shows the true stress strain curves for all the tests, each one characterized by the respective strain rate. 
From experimental data, DIF values can be obtained by fixing the plastic strain value to get both dynamic and quasi static stresses. In this analysis, the plastic strain was fixed in 0.2 . On the other hand, the strain rate values were estimated by averaging them along the test time. Using a logarithmic approach of both sides of equation (7), equation (8), the Cowper-Symonds parameters can be obtained using a linear fitting of the DIF and the average strain rate values, Figure 4 . The quasi static tensile test was performed at the lowest test velocity $(0.1 \mathrm{~mm} / \mathrm{min})$ and was considered as the reference curve. However, this reference curve is also affected by the strain rate sensitivity. For this reason an adjusting routine was necessary to take into account the strain rate sensitivity over the reference test and consequently over all the DIF values, see algorithmic diagram in Figure 5 . This routine is very simple and was performed in commercial spreadsheet software.

$$
\begin{aligned}
& \log (\mathrm{DIF}-1)=\frac{1}{p} \log (\dot{\varepsilon})-\frac{1}{p} \log (C) \\
& \operatorname{Minimize}\left\{\left|\mathrm{DIF}^{i m p}-\mathrm{DIF}^{\text {eval }}\right|\right\}
\end{aligned}
$$

where DIF ${ }^{i m p}$ is the DIF value imposed in the adjusting routine and DIF ${ }^{\text {eval }}$ is the DIF value evaluated using the new parameters. The Cowper-Symonds parameters were estimated with an accuracy of $0.01 \%$ and resulted in $C=24422.58 \mathrm{~s}^{-1}$ and $p=$ 4.6889. On the other hand, if the Cowper-Symonds parameters were evaluated using the traditional calculus procedure, they results in $C=21356.42 \mathrm{~s}^{-1}$ and $p=$ 4.099 so leading to errors in DIF estimative up to $4 \%$ for strain rate range from 0.1 to $1000 \mathrm{~s}^{-1}$. It is worth mentioning that the Cowper-Symonds model was implemented in the numerical model of the optimization routine to evaluate the elasto plastic curve of the material so aiming to include the dynamic sensitivity of the material.

\section{FAILURE}

A failure criterion developed by Törnqvist [20] for numerical modeling of ship collision events was used in this work. The Rice-Tracey-Crockroft-Latham criterion (RTCL criterion) was based in the combination of two ductile failure models: the Rice-Tracey and the Crockcroft-Latham criteria with the aim to cover the full triaxiality range satisfactorily, equation (10). It is because the Crockroft-Latham criterion works adequately to model ductile shear fracture [21], i.e. ductile failure at low triaxialities range and the Rice-Tracey, to model ductile failure due to void growth [22], i.e. ductile failure at high triaxialities range. The Crockroft-Latham criterion was expressed entirely in function of the triaxiality value [23]. Törnqvist [20] validated the RTCL criterion by employing in the numerical modeling of large-scale experiments of diverse ship accident scenarios as the indentation and tearing test of the ship bottom by a truncated cone (to reproduce a stranding scenario) and the lateral collision of a double hull against a bridge pier [20].

$$
D=\int_{0}^{\bar{\varepsilon}_{f}} f\left(\frac{\sigma_{h}}{\bar{\sigma}}\right)_{R T C L} d \bar{\varepsilon}
$$




$$
f\left(\frac{\sigma_{h}}{\bar{\sigma}}\right)_{R T C L}=\left\{\begin{array}{cc}
0 & \frac{\sigma_{h}}{\bar{\sigma}} \leq-\frac{1}{3} \\
\frac{2+2 \frac{\sigma_{h}}{\bar{\sigma}} \sqrt{12-27\left(\frac{\sigma_{h}}{\bar{\sigma}}\right)^{2}}}{3 \frac{\sigma_{h}}{\bar{\sigma}}+\sqrt{12-27\left(\frac{\sigma_{h}}{\bar{\sigma}}\right)^{2}}} & -\frac{1}{3}<\frac{\sigma_{h}}{\bar{\sigma}}<\frac{1}{3} \\
0.6065 e^{\left(\frac{3 \sigma_{h}}{2 \bar{\sigma}}\right)} & \frac{\sigma_{h}}{\bar{\sigma}} \geq \frac{1}{3}
\end{array}\right.
$$

where $D$ is the RTCL failure parameter, $d \bar{\varepsilon}$ is the increment of the equivalent plastic strain, $\frac{\sigma_{h}}{\bar{\sigma}}$ is the triaxiality value, $\sigma_{h}$ is the hydrostatic stress and $\bar{\sigma}$ if the equivalent Von Mises stress. The RTCL failure parameter was obtained by averaging the failure parameters obtained from tests with diverse specimen geometries conceived to induce triaxiality values in the range from 0.0 to 0.6 . These specimens include a plain sample, notched samples and shear samples (with geometries to provoke shear failure), see Figure 6 . The plain sample has a uniform section of $10 \times 0.25$ $\mathrm{mm}$, the notched samples have a minimum section with the same area $(10 \times 0.25$ $\mathrm{mm}$ ) and the shear samples have two symmetric shear sections of $2.5 \times 0.25 \mathrm{~mm}$ each one, Figure 5. The notch radii are $0.25,3.0$ and $10 \mathrm{~mm}$ and the samples were called as Notch 1 Notch 2 and Notch 3 respectively. The angles of the shear sections are $0^{\circ}, 23^{\circ}$ and $45^{\circ}$, measured from the vertical axis, and the samples were also called as Shear 1 , Shear 2 and Shear 3 respectively. This diversity of specimen geometries aimed to induce in the failure section triaxiality values of 0.333 for the plain, from 0.0 to 0.333 for the shear and from 0.333 to 0.666 for the notched geometries as stated in similar researches involving numerical and experimental evaluation of ductile failure [14]. The specimens were fabricated using laser cutting process because it produces a better surface finishing in the cut face and minimal geometric or thermal distortion in the steel sheet material. All experimental tests were performed in an Instron universal testing machine, model 3369, at a cross head speed of $0.0025 \mathrm{~mm} / \mathrm{s}$ and the strain was measured using a $50 \mathrm{~mm}$ clip gage. Figure 7 shows the force displacement curves for all seven specimens. The displacement was measured using a $50 \mathrm{~mm}$ clip gage. A progressive decaying of the peak force in the Notch and Shear samples was observed as the notch radii and shear angles increases respectively.

The numerical models were constructed for every sample geometry using both elasto plastic material model of Voce and strain rate sensitivity model of CowperSymonds with the material parameters estimated as indicated. All the numerical models were created in the Ansys R13 code, the LS-dyna 971 was used as numerical explicit solver and the LS-Pre-Post 4.0 for the post-processing of results. For all specimens, their geometries were modeled using quadrilateral $0.5 \times 0.5 \mathrm{~mm}$ shell elements. As mentioned before, the failure parameter was evaluated as an average of the resulting failure parameters obtained for the seven specimen geometries. Each failure parameter was evaluated in an optimization process, see algorithmic diagram in Figure 8, where the failure parameter $D$ is considered as the variable and the objective function is the minimum difference between the total plastic works of the experimental and numerical tests, equation (11). As the optimization routines took about 3 to 4 cycles to estimate the failure parameter for each geometry, the process was conducted manually. The plastic work was here represented by the area under the force displacement curve. 


$$
\operatorname{Minimize}\left\{\left|\sum_{i=1}^{n} F_{i}^{\exp }\left(\frac{d_{i+1}-d_{i-1}}{2}\right)-\sum_{j=1}^{m} F_{j}^{n u m}(D)\left(\frac{d_{j+1}-d_{j-1}}{2}\right)\right|\right\}
$$

where $\left(F_{i}^{\text {exp }}, d_{i}\right)$ and $\left(F_{j}^{\text {num }}, d_{j}\right)$ are the $i$-th and the $j$-th points of the force displacement curves obtained experimental and numerically, respectively, given that the $F_{j}^{\text {num }}$ is dependent on the RTCL failure parameter. Figure 9 showed the detailed geometry of the ruptured samples after the tests. A perceptible inclination of the ruptured sections can be observed in the notched and shear specimens. Due to the fixed element size of the numerical models, a determined geometry showed more difficulties to adjust to the experimental tests data than the others as the Shear 1. To follow a similar response to the experimental reference, a more refined mesh must be necessary for these tests. However, as stated by previous works of Calle and Alves [17], the failure parameters are sensitive to the mesh size, so the fixed quadrilateral element size of $0.5 \times 0.5 \mathrm{~mm}$ is of mandatory use in this work.

The failure parameters, estimated for all specimen geometries, were presented in Figure 10. The average failure parameter resulted in $D=0.5867$. All tests showed a agreement of its failure parameter values except for the samples Shear 1 and Notch 3. This fact is expected since the Shear 1 sample is unable to achieve a more accurate response because its intricate geometry and the Notch 3 sample showed a different rupture pattern in the numerical modeling, when compared with the other specimens, the crack initiates not in the edge but in the middle of the failure section, in similar form to the plain sample test. The numerical models of the Shear samples showed a high complexity in reproducing shear events, in spite of the average triaxiality in their failure sections remained in the range from 0.0 to 0.333 , the triaxiality value in the first element to fail in the numerical models was always above of 0.333 and the element is located in the edge of the shear sections.

\section{CONCLUSIONS}

The optimization procedure to evaluate the elasto plastic behavior of steel sheet material in quasi static conditions was performed. This evaluation resulted in a more precise mechanical characterization of the material given the good agreement of experimental test with the numerical modeling responses in the uniaxial tensile test. However, it was necessary the implementation of a code to conduct the optimization routine because of the large number of cycles to perform the optimization.

It was demonstrated the importance of considering a non-zero value for the strain rate of the quasi static test used as reference to estimate the parameters of the strain rate sensitivity model as the Cowper-Symonds used in this work. In this respect, a simple adjustment routine used in this work resulted to be suitable for this purpose.

As the evaluation of the elasto plastic behavior of the material needed to include its strain rate sensitivity, the adjustment procedure to obtain the parameters of the strain rate sensitivity model (Cowper-Symonds model) was performed formerly.

The analysis of various specimens to evaluate failure, with diverse triaxialities ranges induced in their ruptured sections, presented similar failure parameters $D$ so 
demonstrating the effectiveness of the RTCL criterion to model diverse failure patterns in a triaxiality range from 0.0 to 0.666 .

All the analysis performed in this research, including the optimization routines to evaluate the elasto plastic behavior, strain rate sensitivity and failure parameters were for steel sheet material and employed numerical models with a fixed shell element size, quadrilateral elements of $0.5 \times 0.5 \mathrm{~mm}$ size. The parameters obtained in this work are particularly limited for modeling thin plate structures built with mild steel sheets and geometry meshed using shell elements with the same size.

\section{ACKNOWLEDGEMENTS}

This work was financially supported by FAPESP (Sao Paulo State Foundation for Research Support, process $n^{\circ}$ 2011/11733-3) and FINEP (Studies and Projects Financing Agency, process $\mathrm{n}^{\circ}$ 01.08.0624.00).

\section{REFERENCES}

[1] Calle, M.A.G. and Alves, M., "Ship collision: A brief survey", International Congress of Mechanical Engineering, Natal, Brazil, (2011).

[2] Hagiwara, K., Takanabe, H. and Kawano, H., "A proposed method of predicting ship collision damage", International Journal of Impact Engineering, Vol. 1, No. 3, pp. 257-279, (1983).

[3] Tabri, K., Määttänen, J. and Ranta, J., "Model-scale experiments of symmetric ship collisions", Journal of Marine Science and Technology, Vol. 13, pp. 71-84, (2008).

[4] Oshiro, R.E., Calle, M.A.G., Mazzariol, L.M. and Alves, M., "Experimental study of scaled $T$ cross-section beams subjected to impact load", International Congress of Mechanical Engineering, Natal, Brazil, (2011).

[5] Mirone, G., "A new model for the elastoplastic characterization and stress-strain determination on the necking section of a tensile specimen", International Journal of Solids and Structures, Vol. 41, pp. 3545-3564, (2004).

[6] Bridgman, P. W., "Studies in large flow and fracture". McGraw-Hill, (1956).

[7] Alves, M. and Jones, N., "Influence of hydrostatic stress on failure of axisymmetric notched specimens", Journal of Mechanics and Physics of Solids, Vol. 47, pp. 643-667, (1999).

[8] Kamaya, M. and Kawakubo, M., "A procedure for determining the true stressstrain curve over a large range of strains using digital image correlation and finite element analysis", Mechanics of Materials, Vol. 43, pp. 243-253, (2011).

[9] Bacha, A., Daniel, D. and Klocker, H., "On the determination of true stress triaxiality in sheet metal", Journal of Materials Processing Technology, Vol. 184, pp. 272-287, (2007).

[10] Pottier, T., Toussaint, F. and Vacher, P., "An inverse method for material parameters determination of titanium samples under tensile loading". International Journal of Metal Forming, Vol. 1, No. 1, pp. 21-24, (2008).

[11] Wisniewski, K. and Kolakowski, P., "The effect of selected parameters on ship collision results by dynamic FE simulations", Finite Elements in Analysis and Design, Vol. 39, pp. 985-1006, (2003). 
[12] Jones, N., "A literature survey on the collision and grounding protection of ships", Ship Structure Committee, (1979).

[13] Wang, G., Spencer, J. and Chen, Y.J., "Assessment of ship's performance in accidents", Marine Structures, Vol.15, pp. 313-333, (2002).

[14] Wierzbicki, T., Bao, Y., Lee, Y-W. and Bai, Y., "Calibration and evaluation of seven fracture models", International Journal of Mechanical Science, Vol. 47, pp. 719-743, (2005).

[15] Calle, M.A.G., Oshiro, R.E., Mazzariol, L.M. and Alves, M., "Structural response of reduced scale naval structures under impact tests", Conference on Structural Nonlinear Dynamics and Diagnosis, Agadir, Morocco, (2014).

[16] Mazzariol, L.M., Calle, M.A.G., Oshiro, R.E. and Alves, M., "Scaling of stiffened panels subjected to impact loading". Iberian-Latin-American Congress on Computational Methods in Engineering, Buenos Aires, Argentina. Mecanica Computacional, Vol. 29, pp. 1275-1289, (2010).

[17] Calle, M.A.G. and Alves, M., "An inverse methodology for tuning material parameters in numerical modeling of mechanical structures", International Symposium of Solid Mechanics, Porto Alegre, Brazil, (2013).

[18] LSTC (Livermore Software Technology Corporation), LS-Dyna keyword user's manual, (2007).

[19] Jones, N., Structural Impact, Cambridge University Press, p. 592, (1997)

[20] Törnqvist, R., "Design of crashworthy ship structures", PhD thesis. Department of Naval Architecture and Offshore Engineering, Lyngby, Denmark. Technical University of Denmark, p. 243, (2003).

[21] Cockcroft, M.G. and Latham, D.J., "Ductility and the workability of metals", Journal Institute of Metal, Vol. 96, pp. 33-39, (1968).

[22] Rice, J.R. and Tracey, D.M., "On the Ductile Enlargement of Voids in Triaxial Stress Fields", Journal of the Mechanics and Physics of Solids, Vol. 17, pp. 201-217, (1969).

[23] Wierzbicki, T. and Werner, H., Report of the Massachusetts Institute of Technology, (1998). 


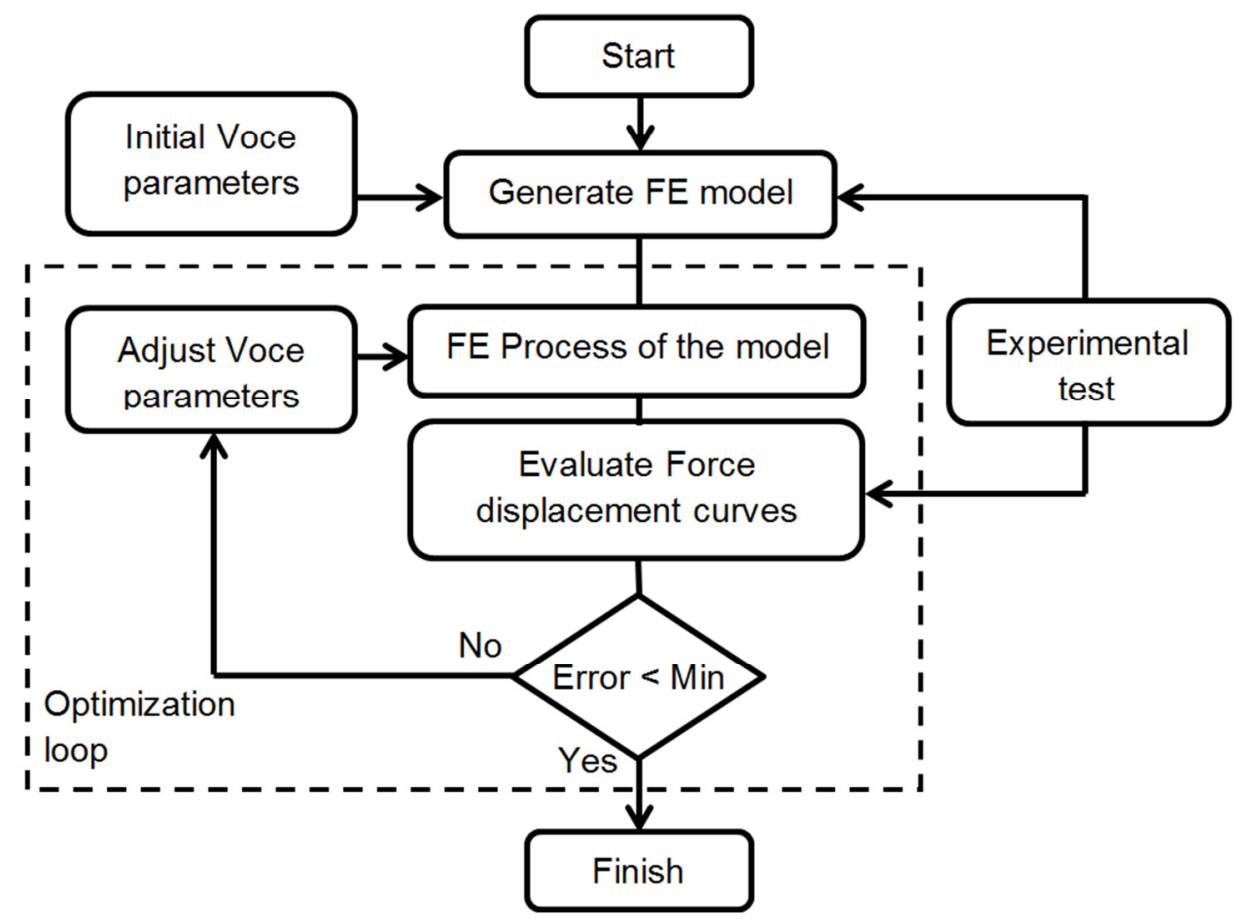

Fig. 1. Diagram of optimization routine to adjust material parameters.

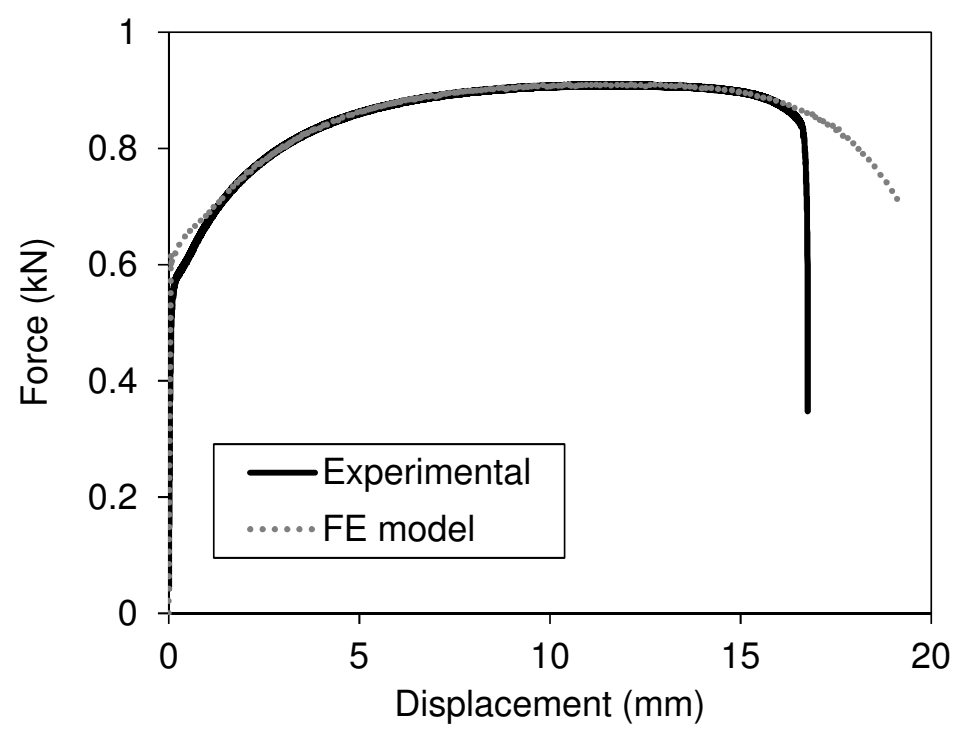

Fig. 2. Experimental and numerical force displacement curves of tensile test in the optimization routine. 


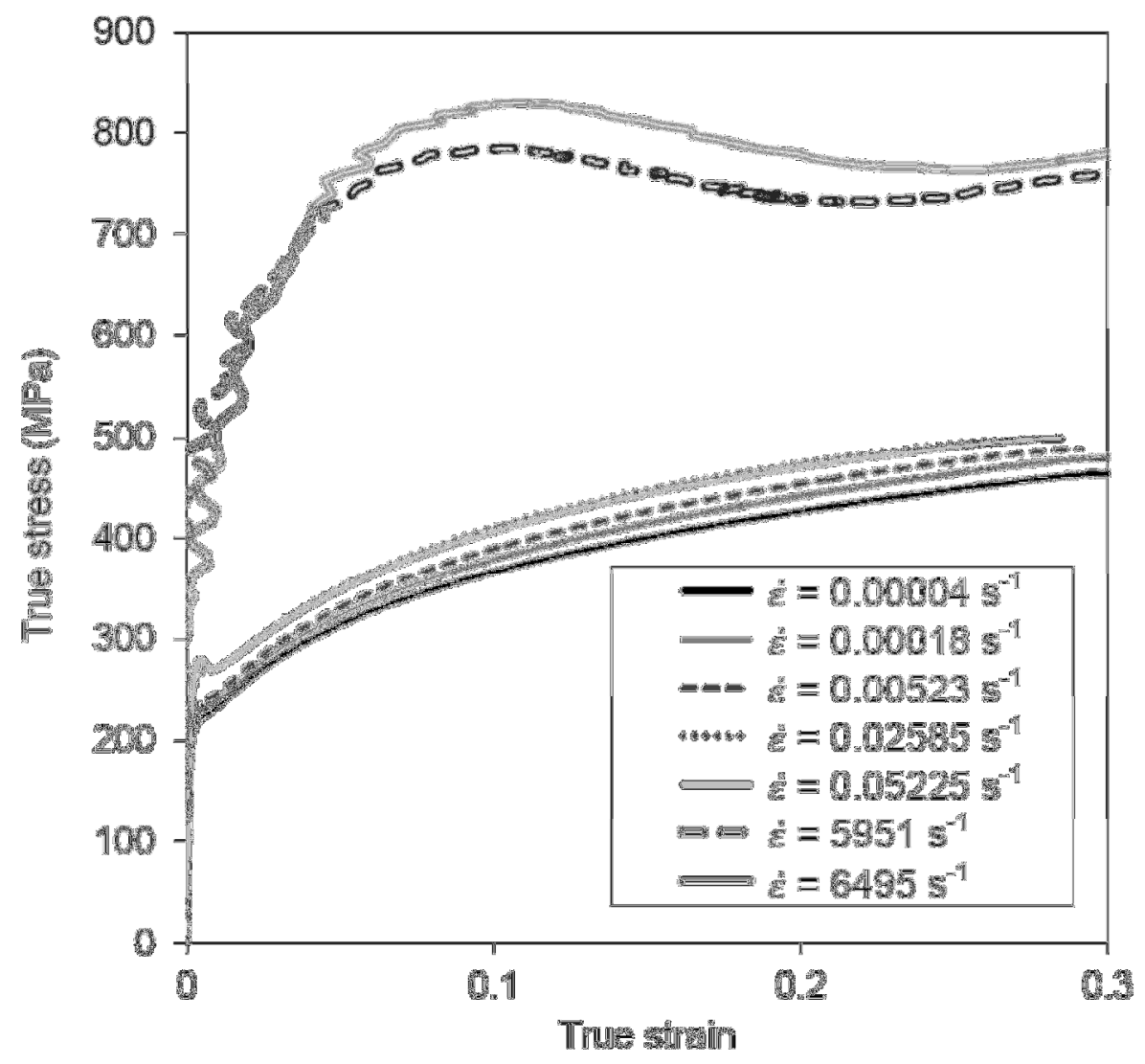

Fig. 3. Experimental true stress strain curves obtained for various strain rates.

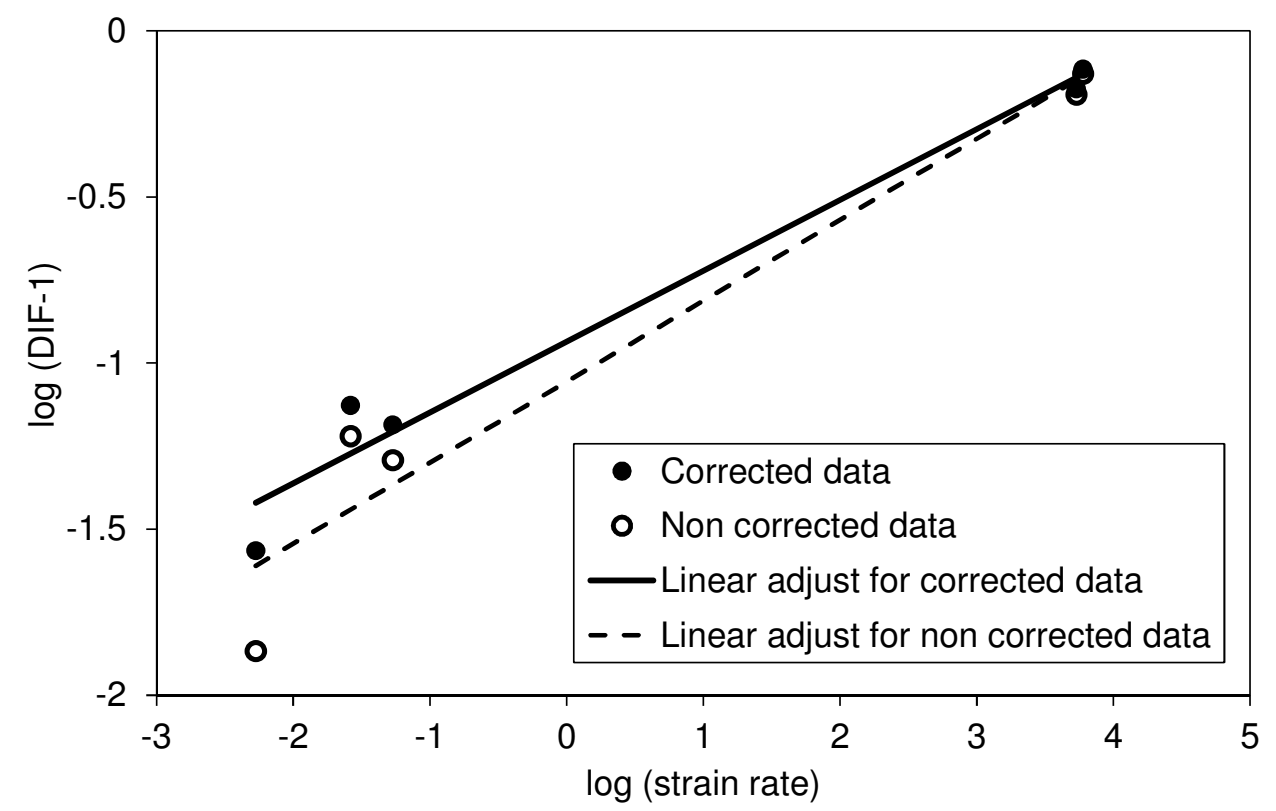

Fig. 4. Logarithmic approach to evaluate strain rate sensitivity parameters using the Cowper-Symonds model. 


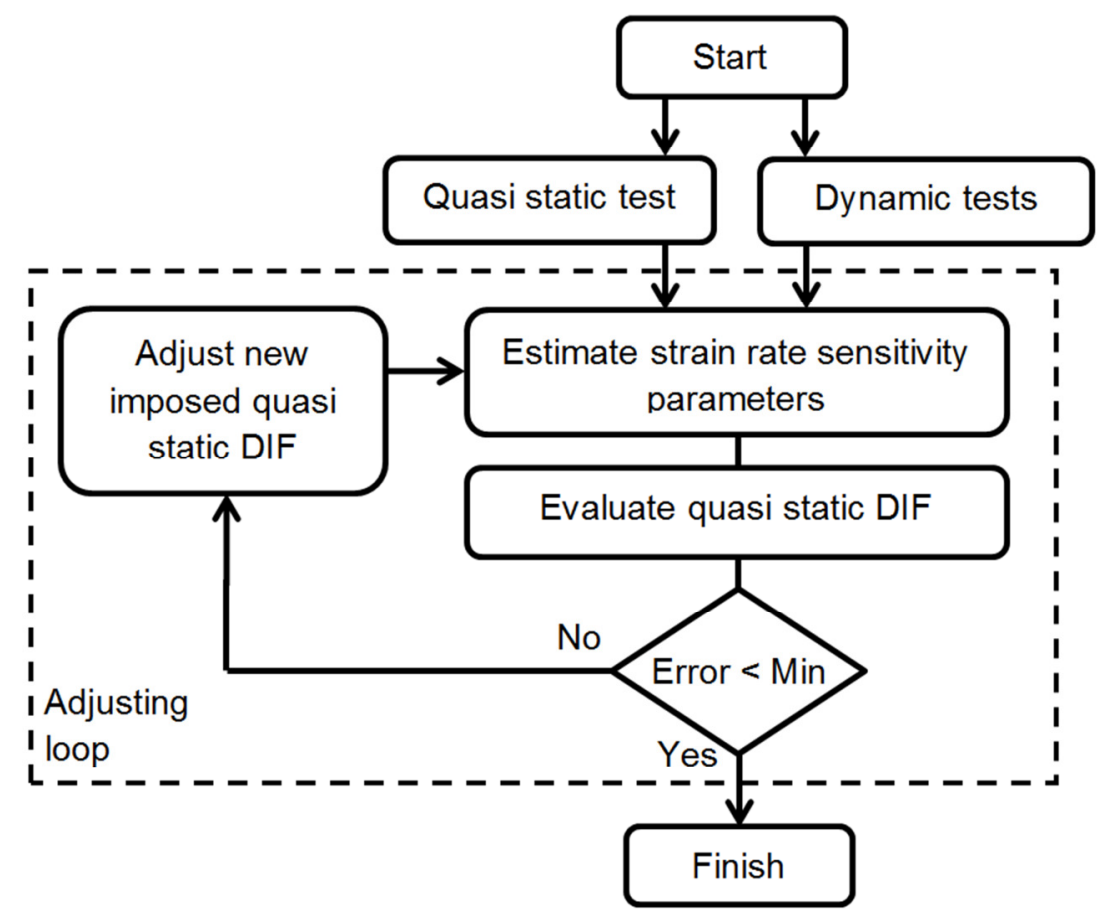

Fig. 5. Diagram of procedure to adjust strain rate sensitivity parameters.
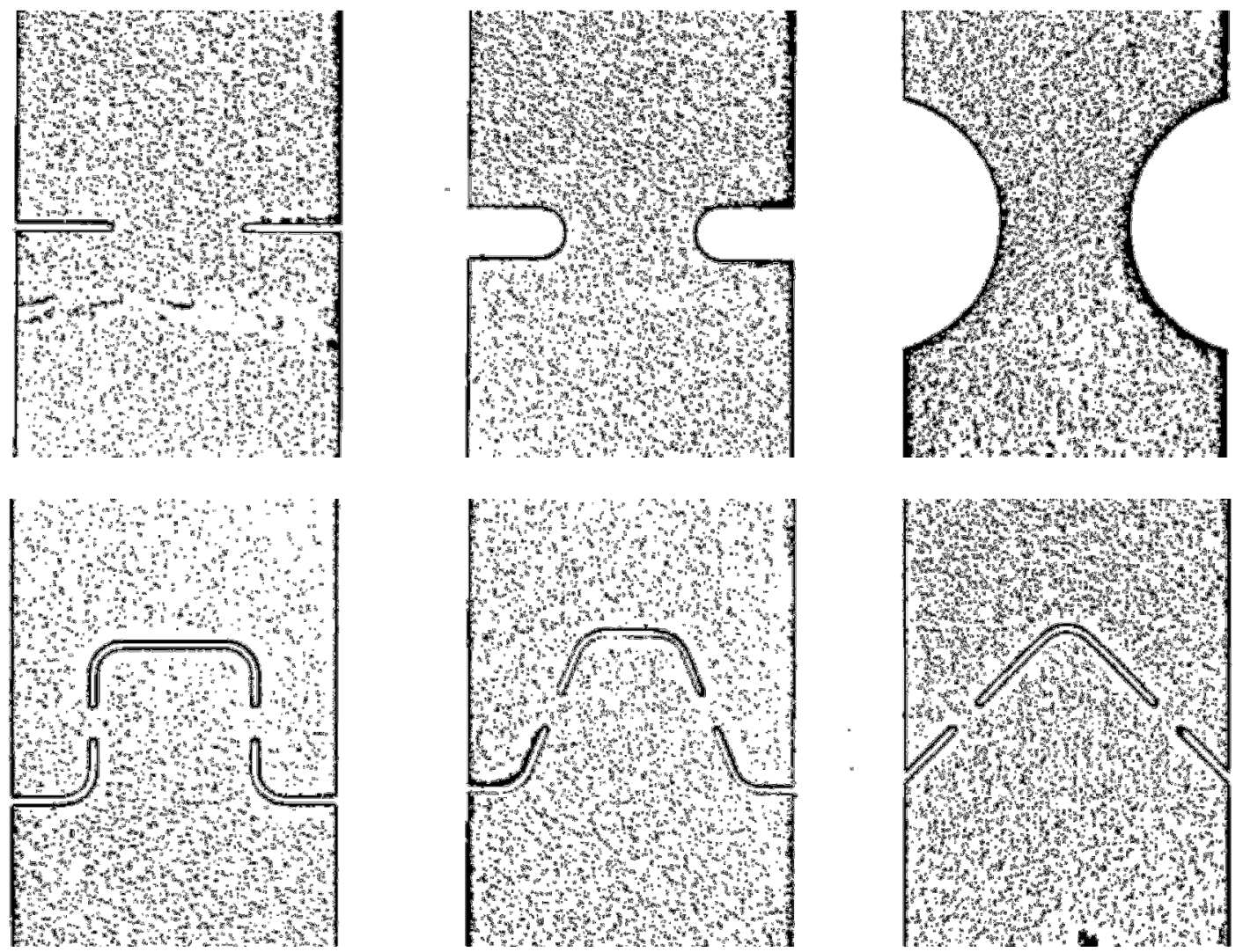

Fig. 6. Details of notched and shear samples before tests. 


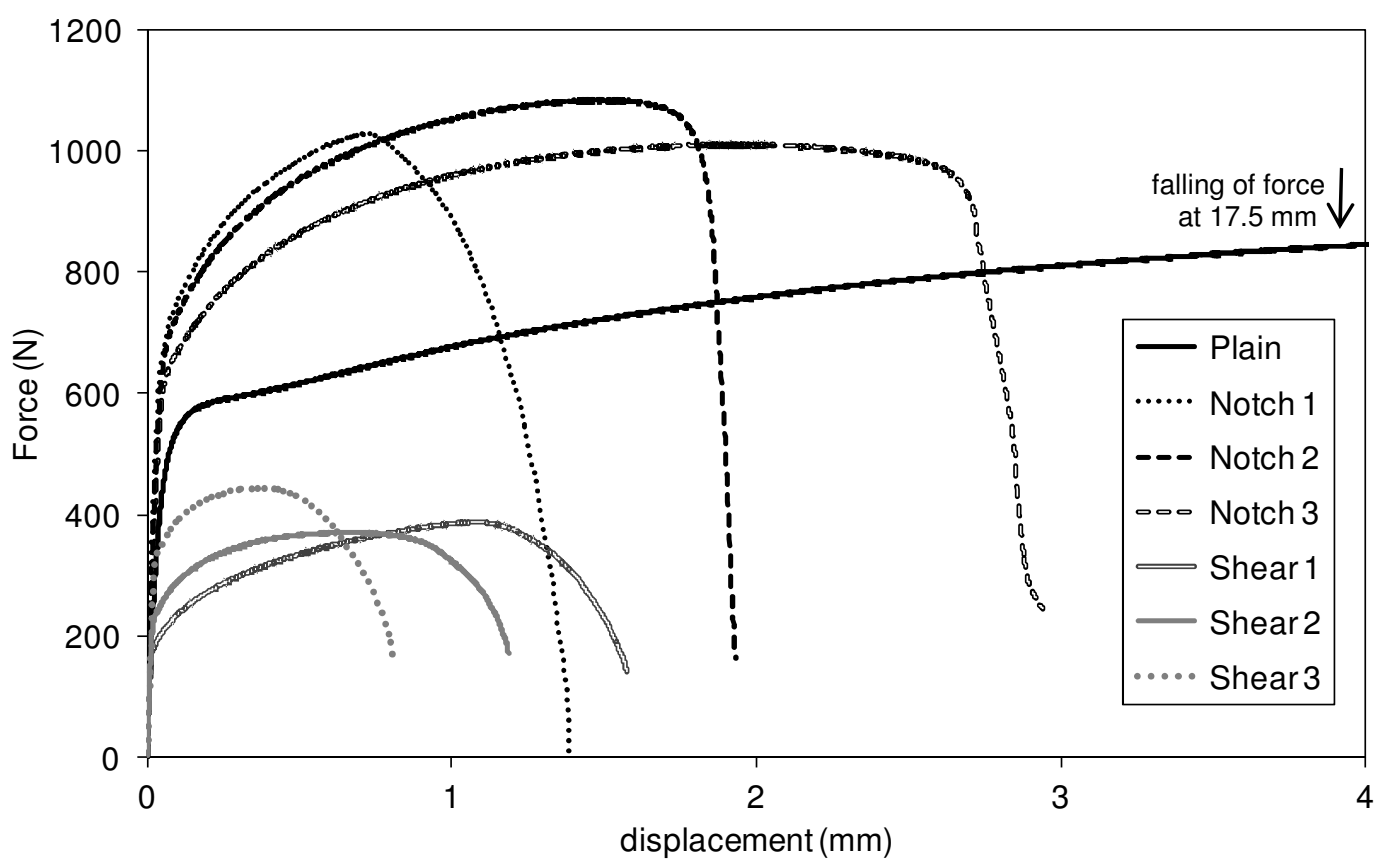

Fig. 7. Force displacement curve for tests in plain, notched and shear samples.

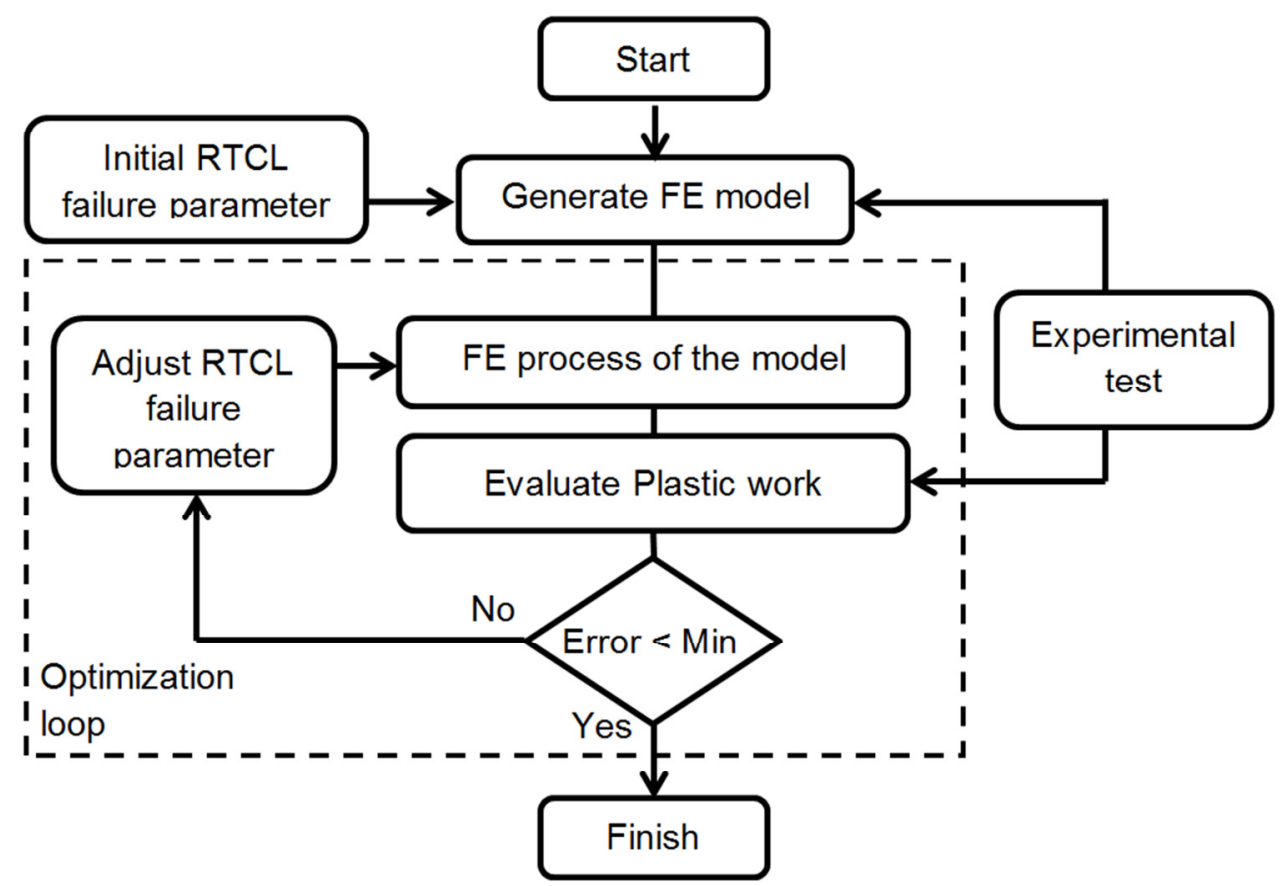

Fig. 8. Diagram of optimization routine to adjust failure parameter. 

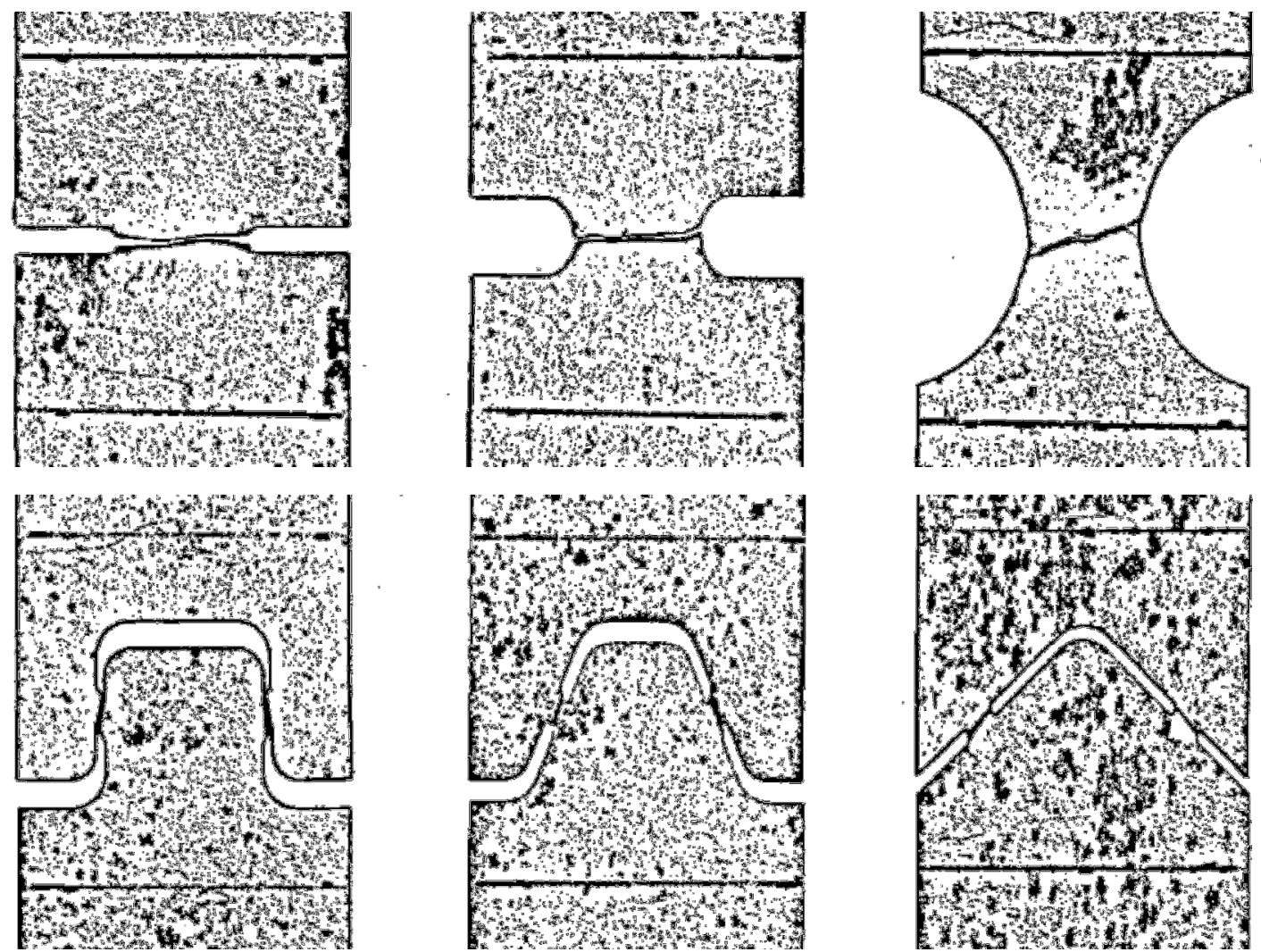

Fig. 9. Detail of ruptured notched and shear samples after tests.

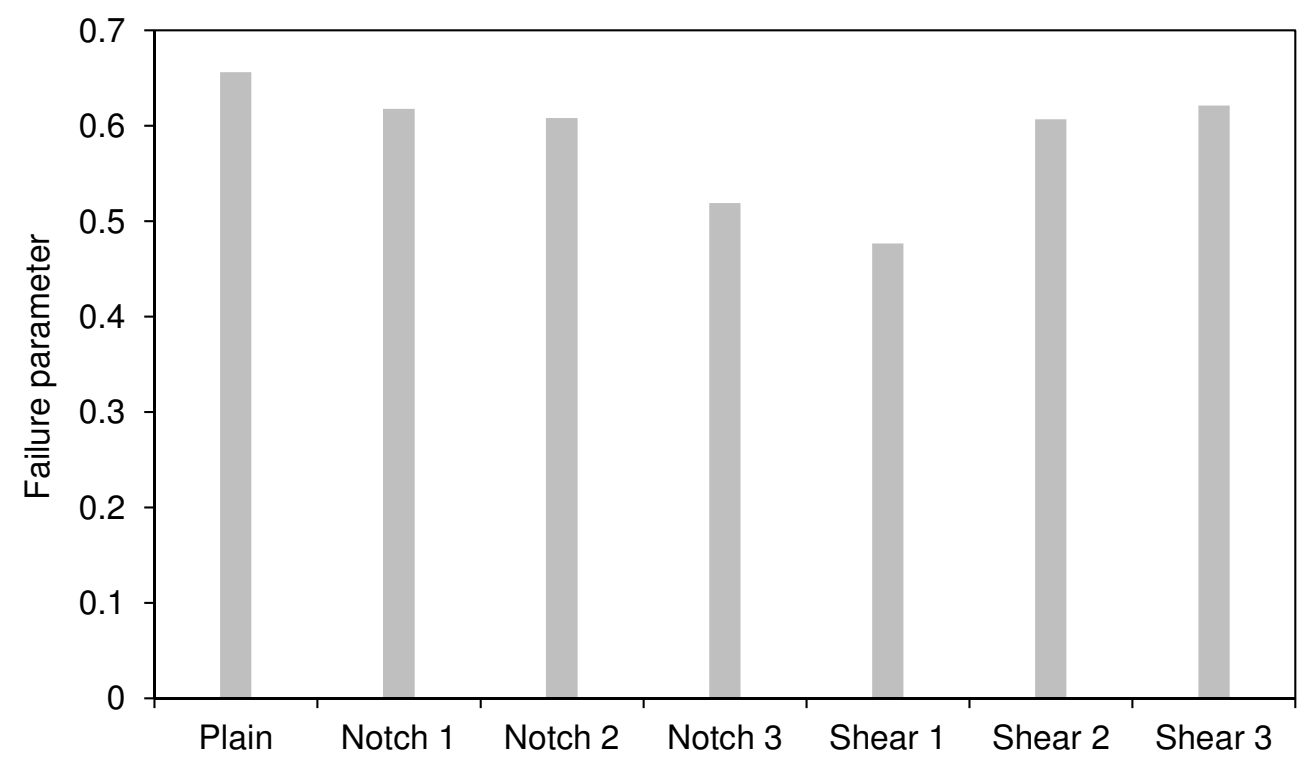

Fig. 10. Failure parameter obtained for all the tests. 